\title{
Carmen Arriagada y \\ la ciudad ausente
}

\section{Leonidas Morales $^{1}$}

Carmen Arriagada escribió sus cartas de amor al pintor alemán Mauricio Rugendas entre la década de 1830 y la de 1850: la primera es de enero de 1836 y la última, de junio de $1851 .^{2}$ Antes de referirme a este período de la historia social chilena cubierto por las cartas, al lugar desde donde tiene lugar la enunciación epistolar de Arriagada, al modo particular de verbalizar su percepción de la vida cotidiana chilena y a los elementos críticos que esa percepción contiene, temas estos últimos desde luego centrales y constantes en el desarrollo de este ensayo, es importante detenerse en las cartas mismas, en su condición de textos y en el efecto que, como tales, producen en la imaginación constructora de su lector actual.

En primer término, no son cartas regidas por la dispersión, reunidas en epistolario por un azar que no las compromete más allá de la reiteración de un estado de la subjetividad, es decir, el estado de enamoramiento. Por el contrario, el epistolario de Carmen Arriagada precipita un fenómeno insólito en la comunicación epistolar del siglo XIX chileno (de hombres y mujeres), y que en el XX lo reeditarán las cartas de amor de Gabriela Mistral y de Violeta Parra. ${ }^{3}$ Quiero decir que el epistolario de Carmen Arriagada no es una simple suma de textos de una mujer enamorada. Desde el primero y hasta el último, estos textos no dejan de activar y escenificar una voz femenina inconfundible, intensa y seductora, que convierte el amor en un código (de origen romántico sin duda) desde el cual o con el cual enuncia un mundo, de remisión biográfica para empezar, pero cuyos signos revelan una capacidad inesperada para sorprender las líneas de sentido que entran en el dibujo de un momento de nuestra historia social y cultural, pero a la vez para abrirse a los límites y a las opciones del sujeto (no sólo femenino) dentro de ese momento, y también, para comprometer en esa aventura del saber al lector 
actual de las cartas, en la medida en que éste se reconozca a sí mismo, como ente de historia, en esa trayectoria social y cultural que desemboca en el presente.

En resumen: las cartas de Carmen Arriagada constituyen, en propiedad, una escritura, entendiendo por tal no simplemente un texto o una mera suma de textos, sino un todo textual, un conjunto de instancias, pliegues o niveles significantes internamente solidarios entre sí, tributarios de un mismo movimiento de sentido discursivo. Algo similar a un proyecto, desplegado pero secreto, enunciado pero no dicho. Una escritura ésta trazada además en zonas limítrofes, sonambulescas, de convivencia, y a veces de indistinción, entre la ficción y la realidad, entre lo vivido y lo imaginado. Siempre portadora de marcas inmediatamente reconocibles como aquellas que definen a una escritura literaria moderna. Más aún: creo que esa voz que enuncia y se enuncia en su escritura, representa, en todo el siglo XIX chileno, y dentro del universo de los géneros referenciales, y no sólo en la carta, un caso único. Es una voz enunciadora que el lector asocia de inmediato, por afinidad (pero sin anular por completo la distancia, la diferencia entre ambas), con la voz del "autor" como función del texto. También en las cartas de Arriagada la voz, como decía Agamben destacando un aspecto fundamental de la definición de autor, es una voz que "se pone en riegos," que al enunciar y enunciarse "se juega" por entero tras una figura de sí, del otro, del tiempo, de las cosas: de la vida situada. ${ }^{4}$

Los historiadores chilenos tienen problemas de lectura cuando se topan con textos como los de Carmen Arriagada. Por una parte, en general no manejan la teoría contemporánea de los géneros del discurso, en particular la de los géneros referenciales a los que pertenecen, junto con la carta, la autobiografía, el diario íntimo, las memorias, la crónica urbana, etc., a pesar de que son géneros a los cuales el discurso historiográfico habitualmente recurre. Si los historiadores no disponen de una teoría que les permita explorar críticamente tales géneros desde su especificidad, o sea, desde el conjunto de propiedades que los identifican, tampoco pueden estar en condiciones de construir el significado no obvio (el obvio no requiere construcción) de sus diversas realizaciones particulares, y así, las lecturas historiográficas de estas realizaciones suelen terminar convirtiendo a los géneros 
en meros depósitos de datos, en "fuentes" de información como material de prueba o de ilustración de tales o cuales tesis. Pero algo todavía peor puede ocurrir: la atribución de un determinado significado a alguna de esas realizaciones a partir del déficit teórico señalado. Así ha ocurrido, recientemente, con las cartas de Carmen Arriagada.

Estoy pensando en el ensayo "Angustia y correspondencia." $\mathrm{Su}$ autor, el historiador chileno Cristián Gazmuri, ${ }^{5}$ intenta dar cuenta en él de lo que a su juicio distinguiría a esas cartas como ejercicio individual de comunicación. Aun cuando en su ensayo habla asimismo de las cartas de amor de Gabriela Mistral, su postura crítica básica la elabora en el comentario de las de Carmen Arriagada. ¿Y cuál sería su propuesta? Como punto de partida, se hace cargo por supuesto de lo que todo lector atento de estas cartas comprueba sin demora: que ellas están gobernadas por una suerte de compulsión, una necesidad imperiosa de volver, una y otra vez, y a lo largo de muchos años, más de quince, a refigurarse la presencia del ausente, la del amado Rugendas, a reponerlo como el tú de su diálogo fantasmal, sin ser un obstáculo insalvable un nuevo amor de Rugendas (el de Clara Contardo, en Valparaíso), ni sus prolongados viajes dentro y fuera de Chile, a otros países latinoamericanos, ni su regreso definitivo a Alemania. Más todavía: Gazmuri también observa con agudeza que muchas cartas suyas no están escritas de una sola vez, que es lo regular, sino "en diferentes días y a diferentes horas," lo que le permite afirmar, con razón, "que el conjunto de la correspondencia de Carmen Arriagada es casi un diario de vida" (Sagrado y Gazmuri 2005, 363).

Sin embargo, Gazmuri no construye opciones de lectura abiertas a partir de lo que él mismo justamente constata. Por ejemplo, y apropósito de su última observación, muy aguda, luego de registrarlo, se desentiende del deslizamiento del género epistolar hacia el campo propio del diario de vida, sin reparar en el interés que reviste la intervención de este género del diario en el género epistolar como marca discursiva reveladora, por un lado, del gesto de durar y continuar en el tiempo de la escritura, y por otro, del anclaje justamente radical del sujeto de la escritura en el contexto histórico de una determinada vida cotidiana, ${ }^{6}$ un sujeto (una voz) que junto con enunciarse en su escritura 
desde tal cotidianeidad, enuncia en ella la crítica de esa misma cotidianeidad. Vuelvo a mi tesis anterior: la ausencia de una teoría de los géneros como sustento crítico, explican tanto el desaprovechamiento de posibilidades de lectura disponibles, como la caída del análisis en una reflexión sin espesor desde el punto de vista crítico, plana e informal, incorporando un lenguaje de resonancias psicoanalíticas, pero en un nivel muy primario, de nula elaboración conceptual.

La lectura de Gazmuri, cuyo objeto inicial, hemos visto, es el retorno de Carmen Arriagada a la carta de amor, un retorno casi obsesivo, adopta, en su desarrollo, la forma de una operación crítica de naturaleza fundamentalmente "reduccionista." Reduce (y rebaja) el sentido de esta insistencia epistolar a una función de catarsis biográfica: es, dice, simplemente "un modo de combatir su soledad y su angustia," una angustia, agrega, de "indudables características patológicas" (358-359). Y en el amor por Rugendas, cree descubrir el síntoma de una verdadera fijación infantil: la del amor por su padre, un hombre de gran prestigio social y político, confundido, a sus ojos, con la ética de lo "digno," pero en la práctica un padre más bien ausente del hogar. Rugendas vendría a ser entonces un sustituto de ese "objeto digno." Así, para ella, "se convertiría en el nuevo hombre ideal," "digno de ser amado," al mismo tiempo que el amor que en ella despierta pasaría a ser un "amor-necesidad." Según Gazmuri, cerrado ya el ciclo de la correspondencia a comienzos de la década de 1850, es decir, sin poder contar en adelante con "el mundo más o menos ilusorio y ficticio de su amor con Rugendas," Carmen Arriagada se precipitaría definitivamente en la "locura" (359, $361,368,370)$, que la acompañaría durante los últimos años de su larga vida (1807-1900). Una propuesta de lectura como ésta no puede ser compartida: desfigura el género de la carta y banaliza literariamente su realización en las cartas de Carmen Arriagada. Si se generalizara la aplicación de este modelo "reductor," acabaría con el arte y la literatura modernos, "reduciéndolos" a meras funciones psicológicas, a síntomas de la angustia, la locura, etc.

Con semejante estrategia crítica, a Gazmuri se le escapan las cartas de Carmen Arriagada como una escritura, como una producción discursiva que elabora una vida cotidiana biográfica, transformando el proceso de elaboración en un orden superior 
de sentido, justamente el orden que le da al texto de las cartas su densidad, su espesor significante, y que le permite al lector actual reconocer la afinidad de la voz que enuncia con la voz propia del "autor," y leer en los signos de la escritura enunciada las claves de una figura histórica de sujeto femenino, ejemplar por la radicalidad de sus términos, la coherencia de su desarrollo, una ejemplaridad desde la cual, por lo mismo, se ilumina una época de la historia social y cultural chilena, pero, y es lo que aquí específicamente importa subrayar, una iluminación desde la vida cotidiana de Carmen Arriagada. Todas estas expectativas, asociadas a la lectura actual de sus cartas de amor, inseparables del verdadero interés que éstas siguen despertando después de un siglo y medio de haber sido escritas, no parecen tener lugar alguno en la propuesta crítica esencialmente reductora de Cristián Gazmuri, ilustrativa de los vacíos teóricos, dije, bastante habituales en los historiadores chilenos cuando se enfrentan a textos literarios. Desde este punto de vista, Alfredo Jocelyn-Holt representa hoy una saludable excepción, aun cuando sus tesis sobre la historia de Chile me parezcan de pronto discutibles y engañosamente alternativas a los enfoques más o menos tradicionales y canónicos. ${ }^{7}$

En un ensayo ya publicado, ${ }^{8}$ he construido una lectura de las mismas cartas que aquí vuelvo a ponerlas como objeto de examen, pero entonces desde otro ángulo de mirada, no disociado sin embargo del que ahora quiero introducir, sino formando parte ambos de lo que podríamos considerar un mismo orden de sentido como orden de enunciación de un sujeto. En ese ensayo anterior, ponía el acento en el tú del diálogo de las cartas, Rugendas, y decía que ese tú, en cuanto objeto de amor, representaba todo lo que el deseo de Arriagada proyectaba como expectativa vital: un modo de sociabilidad, de relación erótica con el cuerpo femenino, de distinguir los valores del espíritu, del arte, de la literatura, y saber reconocer los superiores. En otras palabras, Rugendas representaba para ella un modo de ser cultural cuyas condiciones sociales de existencia quisiera que formaran parte de su entorno, de su vida cotidiana concreta. Pero estas condiciones, que son condiciones de la modernidad, no se daban en su tiempo, en el medio que le tocó vivir, y sólo podían existir, en vidas como las de Carmen Arriagada, a título de ausencia, de imágenes 
fantasmáticas del deseo. A esta ausencia hay que remitir, me parece, todas las noticias sobre la cotidianeidad que le correspondió vivir en la ciudad de Talca (a donde llegó en 1835, luego de una breve estadía en Linares, y en donde murió en 1900), y las reflexiones críticas de que son objeto, reflexiones generalizables, en lo esencial, a toda la sociedad chilena, tal como ésta se ofrecía, en esa primera mitad del siglo XIX, desde el punto de vista del desarrollo de la modernidad desde la Independencia.

Rápidamente se le vuelven familiares al lector algunas constantes del lenguaje epistolar de Arriagada. Por lo pronto, la sintaxis y el léxico de su lenguaje, probablemente habituales dentro de los sectores más cultos de su clase social, en su caso se da siempre abierto al detalle concreto de la vida cotidiana, doméstica y pública, produciendo por esta vía en el lector un "efecto de realidad" seductor. Un lenguaje suelto, ingenioso a ratos, salpicado de palabras o frases en francés (la lengua universal del siglo XIX, hasta las primeras décadas del XX), pero en todo momento lúcido y tributario de una vasta cultura letrada. Los libros no eran fáciles de encontrar todavía en la década de 1830. A ella le llegaban por distintas vías. La más importante era el propio Rugendas, que los conseguía, se los enviaba y le pedía su comentario. No abundarían por esos años los chilenos o chilenas comparables a Arriagada en volumen de lectura y calidad de las mismas. En traducciones o en el original (además del francés dominaba el inglés), leía libros de autores principalmente europeos, asociados al pensamiento científico algunos, a la tradición cultural occidental la mayoría, sobre todo autores de la literatura moderna, desde el barroco, pero con un interés central, privilegiado, por los escritores románticos (alemanes, franceses), con cuya sensibilidad se identificaba, como ella misma lo reconocía. ${ }^{9}$ En su caso al menos, las lecturas no fueron sólo actividades de pasatiempo: de alguna manera hicieron de ella una mujer verdaderamente moderna, sensible a las formas (literarias, artísticas, políticas) de la modernidad europea asociadas a expectativas de libertad o de liberación, en las que, como formas superiores del espíritu, se reconocía.

Son los años, desde las décadas de 1820 y 1830, de la apertura de las clases dominantes de la sociedad chilena a la cultura europea, $y$, dentro de ella, a la francesa como paradigma ${ }^{10}$ (París, "capital 
del siglo XIX," decía Benjamin). Lo cual significó el comienzo de toda una amplia recepción de las modas, la literatura y los gestos culturales, principalmente del movimiento cultural entonces vigente, el romanticismo, y, en otro plano, de modelos políticos e institucionales. Por una parte, salen hacia Europa los primeros representantes o agentes de la nueva República chilena, y lo hijos de la clase social dominante que van a estudiar. Sus contactos con profesionales, intelectuales y artistas, suelen dar origen al viaje posterior al país de algunos de ellos (Feliú). Por otra arte, son también los años del inicio del "neocolonialismo" y de una sensibilidad, la romántica, que, frente a la creciente "racionalización" de la vida cotidiana burguesa (Weber) descubre la seducción de lo "otro" en este sentido, que en su variante más superficial adopta la forma de lo "exótico." A América, como a otros continentes, llegan en gran cantidad, provenientes de distintos países, europeos de diversa condición (agentes comerciales, científicos, artistas, aventureros). Las cartas de Carmen Arriagada registran el paso por Talca de numerosos europeos, y alguno se queda en la ciudad. "Talca está lleno de extranjeros norteamericanos, franceses, ingleses y colombianos. Unos buenos, otros ...quién sabe" (Arriagada 1990, 73).

Carmen Arriagada sin duda fue, en Chile, una representante excepcional de la sensibilidad romántica. En sus cartas ella interioriza (hace suyo) el código del amor romántico que postula la seducción como un puente entre dos polos marcados por la soledad. Desde esa soledad, es decir, desde la distancia de la ausencia del otro, el deseo del amante se define por el deseo de la fusión con el otro como fusión de y con lo semejante. De ahí, por ejemplo, el tópico de las "almas gemelas." Incluso en Arriagada esta relación de almas gemelas (de lo semejante) deriva por un momento a una relación que connota lo incestuoso, aun cuando todo se de en un contexto donde se mezclan el juego y la solidaridad emocional ante el sufrimiento del amado. Sucede cuando muere la hermana de Rugendas, y ante el dolor de éste, ella se ofrece para ocupar en el corazón de Rugendas el lugar de la hermana. La literatura, el arte y la bibliografía crítica en torno a este tema, es decir, el amor en la semejanza, son conocidas y profusas.

Que Carmen Arriagada hable su amor en el código romántico no significa que éste absorba, disolviéndola, toda singularidad 
de ese amor. Significa, por el contrario, que esta singularidad encuentra justamente en el código romántico un modo de enunciarse, o mejor todavía, que el código romántico le permite a ella descubrir y dar forma a la singularidad de su amor. Pero hay aquí una coyuntura biográfica e histórica con consecuencias inmediatas para el tema de este ensayo. No cabe duda: lo que une a Rugendas y a Carmen Arriagada, es decir, el erotismo (sexualidad como cultura) que circula entre ellos, es sólo posible dentro de la cultura moderna, más precisamente, de la cultura moderna del siglo XIX. Pero en Rugendas las condiciones de posibilidad de tal erotismo tienen un anclaje efectivo, real, en la sociedad europea a la que pertenece, la sociedad alemana. No puede decirse lo mismo de Carmen Arriagada. En ella esas condiciones son en gran medida imaginarias: el lenguaje de su amor, la forma misma del sentimiento que verbaliza en código romántico, parecieran ser en buena parte el producto de sus intensas lecturas de escritores románticos, novelistas sobre todo, Dumas de modo entusiasta. Desde esta perspectiva, resulta tentador ver en ella a la primera Madame Bovary de la literatura chilena, al primer caso, temprano incluso, de "mediatización" (otro habla en mí, con mi voz), en el sentido que le da René Girard. ${ }^{11}$

Queda así a la vista la coyuntura biográfica e histórica de la que antes hablaba: al decir que las condiciones de posibilidad del erotismo epistolar de Carmen Arriagada son en gran medida imaginarias, quiero decir que la sociedad a la que ella pertenece, la chilena, y la vida cotidiana que le correspondió llevar, en Talca (no distinta en lo esencial a la de Santiago), no pueden servir de anclaje simplemente porque no son todavía propiamente modernas. De manera pues que su soledad está doblemente determinada: por la ausencia de Rugendas y por la ausencia de una sociedad y una vida cotidiana modernas. Vive el afuera de una soledad que al duplicarse, repitiéndose en dos instancias o escenarios, se hace más intensa, y al permanecer en el tiempo, más desgarrada. Es cierto que en Europa el romanticismo fue él mismo la primera ruptura en la historia de la modernidad con el mundo de la vida cotidiana burguesa, ya en un nivel de "racionalización" (Weber) perturbadora, pero en Carmen Arriagada la mediación literaria (novela, poesía, drama) de esta ruptura sólo puede haber afinado o perfeccionado su sensibilidad perceptiva, 
porque en Chile no había ninguna vida cotidiana moderna o burguesa de esa clase con la que pudiera romperse por saturación: esa vida no existía aún sino bajo la condición de proyectos, de gestos locales y parciales, de formas primarias y por lo demás inestables, en resumen, de formas dentro de un proceso apenas iniciado luego de la Independencia.

¿Qué existía entonces en su lugar, en Chile, en las décadas de 1830 y 1840? Por cierto, y prescindiendo de los cambios a nivel de régimen político-institucional, la misma sociedad que antes, el mismo tejido de la vida cotidiana. Las cartas de Carmen Arriagada son ricas en el registro de detalles, de los pequeños acontecimientos del día tras día. A esta altura no debe sorprender que ese registro no se nos dé meramente como el de un paisaje velado por la ausencia del amado y su efecto inmediato, la soledad, sino bajo la forma de una crítica profunda. Crítica de una vida cotidiana no moderna, heredada, esencialmente colonial. No muy distinta, en este sentido, a la crítica que hace Sarmiento (escritor que Carmen Arriagada admira) en el Faculdo. Es decir, una crítica de las prácticas cotidianas de vida de origen colonial hecha desde una modernidad ausente, desde el horizonte de su puro deseo. En el caso de la chilena, esta segunda ausencia, sumada a la primera, la ausencia del amado Rugendas (dos ausencias intercomunidas), termina por darle a su existencia el marco de un vacío absoluto.

Su crítica es la de la vida cotidiana de la ciudad donde transcurre la mayor parte de su larga vida, Talca, entonces la segunda ciudad del país, de no más de 14.000 habitantes hacia la década de 1830, una cantidad no muy sorprendente si se piensa que la capital, Santiago, en el censo de 1843, aparecía con una población apenas entre 60 y 70 mil habitantes (Feliú 1955, 13). Más allá de estas diferencias en la cantidad de habitantes, interesa metodológicamente una comprobación: lo que Carmen Arriagada observa o forma parte de su experiencia de la vida cotidiana talquina, y se convierte con frecuencia en el objeto de su reflexión crítica, no constituye una excepción ni es tampoco algo repetido pero dentro de límites locales: son observaciones y críticas perfectamente generalizables a la vida cotidiana de toda la sociedad chilena.

Esta misma mirada reflexiva sobre el entorno cotidiano inmediato, está también alerta a los acontecimientos políticos, en 
el país y fuera de él. No a cualquier acontecimiento, sí a aquellos donde su mirada encuentra ocasión para el ejercicio crítico de su insobornable espíritu libertario, la misma libertad con que concibe el sujeto que desea, con que fundamenta su amor. Una crítica, la suya, inseparable de la condena de todo poder de origen ilegítimo, de acto de poder donde ella percibe arbitrariedad, abuso, imposición, o del poder asociado a prácticas del viejo orden colonial. Por ejemplo, no estuvo de acuerdo con la guerra de Portales contra Perú y Bolivia, para ella más bien una maniobra dirigida "por los opresores de Chile" para consolidar un modelo de poder autoritario (el "portaliano"). Ni se alegra del triunfo chileno. Revelando un sentimiento de solidaridad latinoamericanista, le parece "pésimo" que peruanos y bolivianos sean considerados "menos que gallinas" (Arriagada 1990, 187). Del mismo modo, hace una defensa entusiasta de la carta de Francisco Bilbao sobre la "sociabilidad chilena," que provocó la reacción escandalizada de los sectores más conservadores y clericales, y descalifica indignada a quienes protagonizaron la quema pública de los ejemplares del diario donde la carta había sido publicada $(450,453)$.

También toma posición frente a acontecimientos políticos y sociales originados en Europa, de los que se informa por la prensa. En términos que nos resultan muy actuales (piénsese sólo en la política de Estados Unidos en Irak, Afganistán y en otros lugares del mundo), se refiere a los discursos ideológicos, a las estrategias de política internacional del poder de las naciones de mayor desarrollo de Europa, a las que llama irónicamente "las señoras potencias": "Las gacetas inglesas me han entretenido algo; pero también me han confirmado en la idea de que las señoras potencias europeas sacan la espada para defender los derechos de las otras naciones cuando les conviene a sus miras particulares y jamás por la justicia de su causa como lo quieren hacer creer" (285). Por último, informada de los sucesos de 1848 en Francia, conocidos como la rebelión popular de la Comuna, se identifica con el sentido de lo que ella llama una "revolución," la que debería llevar a "igualizar un poco la condición física y moral de las clases pobres con las gentes ricas" (517).

La crítica del poder que leemos en las cartas de Carmen Arriagada, responde a una posición discriminada de modernidad. La ética social y el espíritu libertario de esta escritora (influido, es 
muy probable, por la proximidad de las luchas de la Independencia, incluso por el rol de su padre en esas luchas, y de sus propios recuerdos biográficos de algunos de sus protagonistas, como San Martín) representan efectivamente elecciones dentro del campo de opciones de la modernidad. La modernidad, desde el comienzo, nunca fue homogénea. Siempre se desarrolló entre dos polos, paralelos y de complejas relaciones entre sí: el humanista y libertario, de un lado, y el tributario de los intereses burgueses del capital, del otro. ${ }^{12}$ Pero esta mujer que en sus cartas se define políticamente en los términos dichos, es un sujeto con vida cotidiana. En lo que sigue, trataré de poner a la vista algunos aspectos de su conflictiva inserción, como sujeto de modernidad, en la vida cotidiana de esos años en una ciudad como Talca. Dije antes que es una inserción desde un afuera: desde su no reconocimiento cultural, existencial, en el orden de las rutinas y la gestualidad del vivir de todos los días. Un orden, también lo adelanté, de origen colonial. La crítica de este orden es constante en las cartas. Y es constante también el sentimiento de soledad dentro de ese orden.

La cotidianeidad propiamente moderna sólo puede ser urbana. La historia de la modernidad es pues al mismo tiempo la historia de la ciudad. En el caso europeo, esa historia ha sido construida desde comienzos del siglo XX, destacando las grandes transformaciones sufridas por el paradigma urbano, como las que inicia en Francia a mediados del siglo XIX el barón de Haussmann, o las que señala el paso de la modernidad a la posmodernidad desde la década de 1980. En cualquier caso, hay en el espacio de la ciudad un componente central: la calle. La calle como un lugar intransitivo, al que se va y en el que se está, donde se suceden las tiendas y vitrinas, los cafés, donde el transeúnte es espectador y espectáculo, donde se producen los encuentros o, más bien, los desencuentros. ${ }^{13}$ Una calle así (y una ciudad que la haga posible) sólo comenzará a tener de verdad existencia en Chile hacia las dos últimas décadas del siglo XIX, pero casi como enclave. Otras ciudades latinoamericanas, como Buenos Aires, y a distintos ritmos, vivirán este mismo proceso de urbanización, y serán los espacios de vida cotidiana que darán sustento al nacimiento y desarrollo del primer movimiento poético de verdad moderno: el "modernismo" de Rubén Darío. ${ }^{14}$ 
¿Qué era la ciudad chilena en las décadas de 1830-1850 como escenario de vida cotidiana moderna? Concretamente, ¿qué era la ciudad de Talca? Había sí indicios de creación de una nueva ciudad que hiciera posible la calle en el sentido descrito. No voy a referirme a la ciudad de Santiago, donde los indicios son otros en magnitud, frecuencia, anticipación en el tiempo, y también de resultados más estables, pero que no modifican, en el período, la situación básica de carencias de modernidad, de intentos de transición reciente desde un orden colonial. Sin duda, en Talca esos indicios son menores y sin duda más precarios, pero tal vez por eso mismo hacen visible mejor, y sin atenuantes, lo aún dominante. Carmen Arriagada, en sus cartas, no sólo informa de pequeñas empresas "modernizadoras," sino que ella misma también participa de algunas. Por ejemplo, habla con entusiasmo de la apertura de un teatro en la ciudad, aunque luego dará cuenta de su posterior languidecimiento, de la partida de los actores, por desinterés del público. También anuncia el interés de amigos suyos en la instalación de una escuela para niños, sobre todo, agrega ella, para "niñas," consciente de la posición de desventaja y subordinada, de la mujer. El proyecto de esta escuela finalmente no prosperó. Sí tuvieron éxito la traída de una imprenta y la publicación de un periódico, El Alfa, que tuvo varios números y en el que ella misma colaboró, no con crónicas sino con traducciones.

Como actividad cotidiana, hay una en que Carmen Arriagada participa y que viene a ser algo así como una célula comunitaria, de sociabilidad moderna, pero una célula cerrada sobre sí misma, sin un cuerpo social al que integrarse: la tertulia. ${ }^{15}$ Se reúne en su casa con un pequeño grupo, siempre los mismos, a conversar de temas de interés compartido y asociados, por noticias, por lecturas, al horizonte de la modernidad. Hay una segunda actividad cotidiana, menos social, más privada, más solitaria también, portadora de signos luminosos desde el punto de vista de su proyección social. Cuando la tertulia suspende su tiempo, Carmen Arriagada, devuelta al tiempo dominante, lee y escribe. $Y$ hay algo que ella realiza con particular gusto y constancia: teje. Lo hace en forma tan regular y poniendo tal interés, que no resulta forzado asociar el acto de tejer con el de la escritura (otro tejido) de sus cartas: en ambos actos hay una artesanía, una manualidad (escribir y tejer) puesta en juego por una imaginación 
que busca vías por donde salir del presente, ir más allá de él, superarlo, trascenderlo. Una imaginación pues abierta al futuro, a la modernidad de un deseo, o al deseo de una modernidad. Tejer, escribir: fantasmalizar el deseo, figurar la ausencia.

Metonimia de la ciudad moderna, la calle, esa a la que me refería más arriba, es decir, ese escenario donde toma forma concreta y se exhibe públicamente la vida cotidiana, donde se dan los encuentros, los desencuentros, también los estallidos sociales, lugar de la experiencia de un tiempo y de un espacio, los modernos, en permanente fuga y transformación, esa calle, para Carmen Arriagada como para la sociedad chilena de su momento, simplemente es una ausencia absoluta. Habrá que esperar a Joaquín Edwards Bello y sus crónicas para asistir a la presencia de esa calle y de la ciudad que la hace posible. La calle de Carmen Arriagada pertenece todavía, y plenamente, al orden colonial, a su tiempo y a su efecto de inmovilidad. En una carta de enero de 1840, se muestra a sí misma en esta calle: "Paso sola todo el día; coso, bordo y leo cuando mi salud me lo permite; salgo a dar por las tardes una vuelta de dos cuadras y me siento después a tomar el fresco en la puerta de calle, costumbre impropia pero que es muy cómoda para las personas que no pueden salir y están solas" (515).

Esa decisión suya de salir a sentarse en la puerta de calle de su casa a "tomar el fresco," ella la considera "impropia" (¿de su clase social?, ¿de su cultura?, ¿de ambas?, ¿excusa para un gesto que pudiera resultarle concesivo al destinatario de la carta?), pero también se anticipa a decir que es una "costumbre," es decir, algo de reiteración tradicional. Lo que aquí, en este contexto, equivale a decir: colonial. De eso es de lo que, sentada ahí, frente a la calle, es testigo: del transcurrir de un tiempo que, como experiencia cotidiana, no puede sino exacerbarla porque no la domicilia sino que la margina, la expulsa, la lleva a sentirse habitando un "vacío."

Son muchas las cartas en que a la soledad de la ausencia del amado Rugendas, se suma, intensificándola, la soledad de esta otra ausencia: el vacío de vida cotidiana moderna. Hay sí algunas cartas en que el sentimiento de este vacío toca la escritura con particular dramatismo. Por ejemplo, en una carta de 1836, exasperada por la inmovilidad de ese tiempo colonial, escribe: 
"A veces deseo que hubiera un trastorno general en el país, sea por revolución de los hombres o de la naturaleza; me falta movimiento, tengo necesidad de alguna cosa que me conmueva mucho; aunque estoy cierta que la agitación me aniquilaría" (51). En otra carta posterior, de agosto de 1837, lleva su exasperación a una formulación mucho más absoluta aún: "casi no hay cómo seguir una carta, falta de materia. Dios mío, cómo puede uno vivir en estos pueblos! es para morir de fastidio. Vea $\mathrm{Vd}$., mi querido amigo, en todas partes es corta, es escasa la vida; sólo aquí está de más. Oh! sí de más, no sabe uno como llenarla" (99).

\section{Notas}

1 Doctorado en Filosofía con mención en Literatura. Profesor de Literatura Chilena e Hispanoamericana en universidades de Chile, Estados Unidos y Venezuela (donde vivió exiliado), enseña actualmente en la Universidad de Chile. Junto a numerosos ensayos de crítica literaria y cultural en revistas universitarias chilenas y extranjeras, ha publicado asimismo varios libros.

2 Estas cartas han sido editadas por Oscar Pinochet de la Barra con el título de Carmen Arriagada. Cartas de una mujer apasionada. Santiago de Chile: Editorial Universitaria, 1990.

3 Véase mi libro Carta de amor y sujeto femenino en Chile. Siglos XIX y XX. Santiago de Chile: Editorial Cuarto Propio, 2003.

4 Giorgio Agamben, "El autor como gesto." Profanaciones. Trad. Flavia Costa y Edgardo Castro. Buenos Aires: Adriana Hidalgo Editora, 2005, pp. 79-94.

5 El ensayo de Gazmuri aparece en el primer tomo colectivo de la Historia de la vida privada en Chile. El Chile tradicional. De la Conquista a 1840. Bajo la dirección de Rafael Sagredo y Cristián Gazmuri. Santiago: Aguilar Chilena de Ediciones, 2005, pp. 355374.

6 El diario de vida como género referencial tiene, entre otras propiedades, la de su máxima cercanía con la menudencia de lo cotidiano. Véase Maurice Blanchot, "El diario íntimo y el relato." En El libro que vendrá. Trad. Pierre de Place. Caracas: Monte Avila Editores, 1992, pp. 207-212.

7 En uno de sus libros, El peso de la noche. Santiago: Editorial Planeta, 1996, aborda justamente el período de la historia chilena al que pertenecen las cartas de Carmen Arriagada.

8 "Carmen Arriagada: la carta como espacio de construcción del objeto del deseo." En mi libro Carta de amor y sujeto femenino en Chile. 
Siglos XIX y XX. Santiago de Chile: Editorial Cuarto Propio, 2003, pp. 17-36.

9 Sobre las lecturas de Carmen Arriagada y la circulación del libro en su tiempo, ver Susana Zanetti, La dorada garra de la lectura. Rosario (Argentina), Beatriz Viterbo Editora, 2002. El capítulo sobre Arriagada ("Carmen Arriagada, una lectora romántica"), pp. 61-1006.

10 Guillermo Feliú Cruz describe la sociedad chilena de esos años, sus casas, sus gustos estéticos, sus déficits de modernidad, sus políticas de modernización, tomando como pie los personajes sociales, políticos y culturales retratados por Monvoisin, en La sociedad chilena que conoció Monvoisin. Santiago, Editorial Universitaria, 1955. Un folleto de 20 páginas.

11 René Girard desarrolla su concepto de "mediatización" como una forma de alienación, analizando personajes novelesco como Don Quijote y Madame Bovary, en su libro Mentira romántica y verdad novelesca. Trad. Guillermo Sucre. Caracas: Universidad Central de Venezuela, 1963, pp. 7-40.

12 Hardt, Michael y Negri, Antonio. Imperio. Trad. Alcira Bixio. Buenos Aires: Editorial Paidós, 2002.

13 Lefebvre, Henri. La revolución urbana. Trad. Mario Nolla. Madrid: Alianza Editorial, 1980, pp. 24-28.

14 Ver sobre la historia de la ciudad latinoamericana, el estupendo libro de José Luis Romero, Latinoamérica: las ciudades y las ideas. Buenos Aires, Siglo XXI Editores, 2004 ( $1^{\text {a }}$ ed., 1976).

15 Entre los trabajos existentes dedicados a las tertulias y salones en Chile, destaco el de Hernán Godoy Urzúa, "Salones literarios y tertulias intelectuales en Chile, trayectoria y significación sociológica." En Fundación Mario Góngora, Formas de sociabilidad en Chile 1840-1940. Volumen colectivo. Santiago, Editorial Vivaria, 1992, pp. 137-151.

\section{Bibliografía}

Agamben, Giorgio: "El autor como gesto." Profanaciones. Trad. Flavia Costa y Edgardo Castro. Buenos Aires: Adriana Hidalgo Editora, 2005, 79-94.

Arriagada, Carmen. Carmen Arriagada. Cartas de una mujer apasionada. Ed. Oscar Pinochet de la Barra. Santiago de Chile: Editorial Universitaria, 1990.

Blanchot, Maurice. "El diario íntimo y el relato." El libro que vendrá. Trad. Pierre de Place. Caracas: Monte Avila Editores, 1992, 207-212.

Feliú Cruz, Guillermo. La sociedad chilena que conoció Monvoisin. Santiago de Chile: Editorial Universitaria, 1955. 
Girard, René. Mentira romántica y verdad novelesca. Trad. Guillermo Sucre. Caracas: Universidad Central de Venezuela, 1963, 7-40.

Godoy Urzúa, Hernán. "Salones literarios y tertulias intelectuales en Chile, trayectoria y significación sociológica." Formas de sociabilidad en Chile 1840-1940. Ed. Fundación Mario Góngora. Santiago de Chile: Editorial Vivaria, 1992, 137-151.

Hardt, Michael y Negri. Antonio. Imperio. Trad. Alcira Bixio. Buenos Aires: Editorial Paidós, 2002.

Jocelyn-Holt, Alfredo. El peso de la noche. Santiago: Editorial Planeta, 1996.

Lefebvre, Henri. La revolución urbana. Trad. Mario Nolla. Madrid: Alianza Editorial, 1980, 24-28

Morales, Leonidas. "Carmen Arriagada: la carta como espacio de construcción del objeto del deseo." Carta de amor y sujeto femenino en Chile. Siglos XIX y XX. Santiago de Chile: Editorial Cuarto Propio, 2003, 17-36

- Carta de amor y sujeto femenino en Chile. Siglos XIX y XX. Santiago de Chile: Editorial Cuarto Propio, 2003.

Romero, José Luis. Latinoamérica: las ciudades y las ideas. 1976. Buenos Aires: Siglo XXI Editores, 2004.

Sagrado, Rafael y Gazmuri, Cristián, dir. Historia de la vida privada en Chile. El Chile tradicional. Vol. I: De la Conquista a 1840. Santiago de Chile: Aguilar Chilena de Ediciones, 2005, 355-374

Zanetti, Susana. "Carmen Arriagada, una lectora romántica." La dorada garra de la lectura. Rosario, Argentina: Beatriz Viterbo Editora, 2002, 61-1006. 